\title{
Abundance and Star Formation Determinations in MARK galaxies from SDSS Spectra
}

\author{
M. V. Gyulzadyan ${ }^{1 *}$, A. M. Mickaelian ${ }^{1}$, H. V. Abrahamyan ${ }^{1}$, G. A. \\ Mikayelyan $^{1}$, G. M. Paronyan ${ }^{1}$ \\ ${ }^{1}$ NAS RA V. Ambartsumian Byurakan Astrophysical Observatory (BAO), Armenia \\ *E-mail: mgyulz@yahoo.com
}

\begin{abstract}
We analyse the oxygen and nitrogen abundance and specific star formation rates (sSFR) in Markarian galaxies from Sloan Digital Sky Survey (SDSS) spectra. The Data Release 7 (DR7) of SDSS contains photometric data for more there 1000 and spectral information for more then 700 Markarian objects. The Mrk sample has played a central role in the task of distinguishing between the astrophysical different types of phenomena that occur in AGNs. In the course of the Markarian survey, more than 200 Seyfert galaxies, and hundreds of starburst, blue compact, and H II galaxies were discovered. The Markarian survey remains perhaps the best-known source of such objects in the local universe. We have measured their line fluxes and derived the $\mathrm{O}$ and $\mathrm{N}$ abundances using recent calibrations. We have compared the oxygen and nitrogen abundances derived from global emission-line Sloan Digital Sky Survey (SDSS) spectra of galaxies using (1) the Te method and (2) two recent strong-line calibrations: the ON and NS calibrations. The behavior of the $[\mathrm{N} / \mathrm{H}]$ ratio in under abundant regions gives strong support to a partially primary origin of nitrogen. The star formation rate (SFR) is one of the main parameters used to analyse the evolution of galaxies through time. In the local Universe, the $\mathrm{H}_{\alpha}$ luminosity derived from IFS observations can be used to measure SFR, at least in statistically significant, optically-selected galaxy samples, once stellar continuum absorption and dust attenuation effects are accounted for.
\end{abstract}

Keywords: galaxies, Markarian galaxies, spectra.

\section{Mark galaxies}

The First Byurakan Spectral Sky Survey (FBS), also commonly known as Markarian Survey, was initiated in 1965. It was the first systematic objective-prism search for galaxies with strong ultraviolet (UV) continuum 
emission. The observations were carried out with Byurakan Astrophysical Observatory (BAO, Armenia) $1 \mathrm{~m}$ Schmidt telescope equipped with a lowdispersion $\left(2500 \mu\right.$ at $\mathrm{H}_{\beta}$ and $1800 \mu$ at $\left.\mathrm{H}_{\beta}\right) 1.5^{\circ}$ objective prism. It was mostly used with Kodak IIa-F plates to detect galaxies with excess UV radiation (UVX, UV-excess). The survey consisted of 1133 fields (each having $4^{\circ} \times 4^{\circ}$ size) and covered $17,056 \mathrm{deg}^{2}$ north of $-15^{\circ}$ declination, excluding some regions within $15^{\circ}-20^{\circ}$ of the Galactic plane. It was completed in 1978 and published in a series of 15 papers including 1500 UVX objects (Markarian et al. 1981 and references therein).

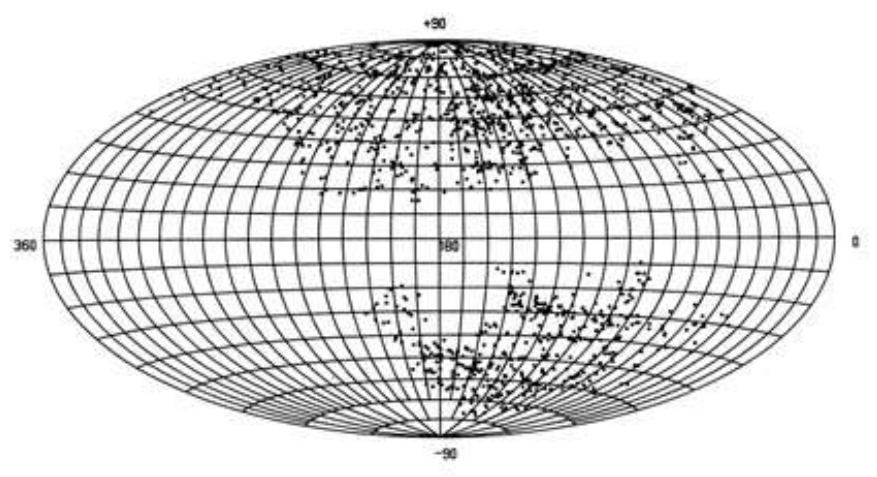

Figure 1: An all sky Hamer-Aitof projection of the 1500 Mark galaxies in Galactic coordinates $(1, \mathrm{~b})$

The Mrk sample has played a central role in the task of distinguishing between the astrophysical different types of phenomena that occur in AGNs. For example, the original stratification of Seyfert galaxies into two types by Khachikian and Weedman (1971) was based largely on measurements of emission-line widths in spectra of a small number of galaxies discovered very early in Mrk survey. Later among MRK galaxies were discovered LINERS (Heckman 1980), galaxies with spectra that resemble HII regions (French 1980) (HII galaxies). Active galactic nuclei which appear to be the result of a short-lived outburst of star formation have become known as starburst galaxies (Weedman et al. 1981).

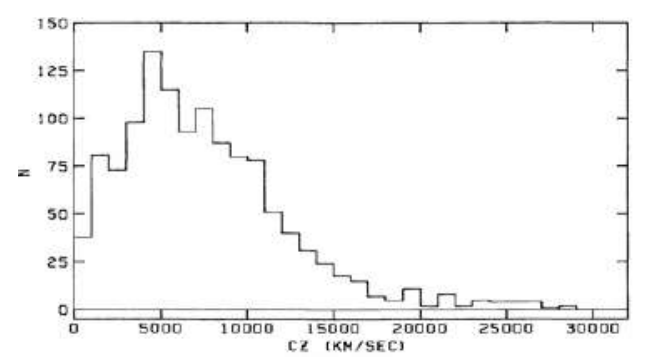

Figure 2: Velocity distribution of Mark galaxies. 


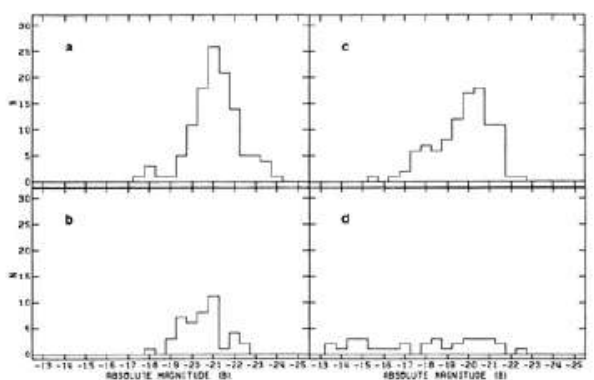

Figure 3: a)distribution of Sy1 \& intermediate galaxies, b)distribution of Sy2 galaxies, c)distribution of SB galaxies, c)distribution of HII galaxies

The $70 \%$ region of the sky covered by the FBS is represented in the SDSS catalog (Sloan Digital Sky Survey; http://www.sdss.org). The Data Release 7 (DR7) of SDSS contains photometric data for more there 1000 and spectral information for more then 700 Markarian objects. The following emission lines are observed in the spectra of these galaxies: $\mathrm{H}_{\alpha}, \mathrm{H}_{\beta},[\mathrm{OIII}] \lambda \lambda 4959 \AA$, $5007 \AA,[\mathrm{NII}] \lambda \lambda 6548 \AA, 6584 \AA,[\mathrm{OII}] \lambda \lambda 3726 \AA, 3729 \AA,[\mathrm{OI}] \lambda 6300 \AA,[\mathrm{SII}] \lambda \lambda$ $6717 \AA, 6731 \AA$, etc. The majority of starburst nuclei have [OIII] $\lambda \lambda 4959$, 5007 emission-line widths less than $250 \mathrm{~km} / \mathrm{s}$ full width at half-maximum (FWHM), with a median FWHM of $140 \mathrm{~km} / \mathrm{s}$. This is to be compared with the median FWHM of Sy [OIII] emission lines, $375 \mathrm{~km} / \mathrm{s}$ for Sy 1 galaxies and $510 \mathrm{~km} / \mathrm{s}$ for Sy 2 galaxies.

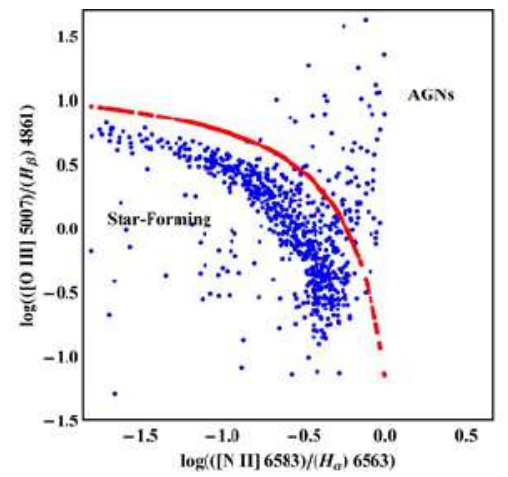

Figure 4: AGN selection in the Lee diagram (2008) for Mark galaxies from SDSS DR7 

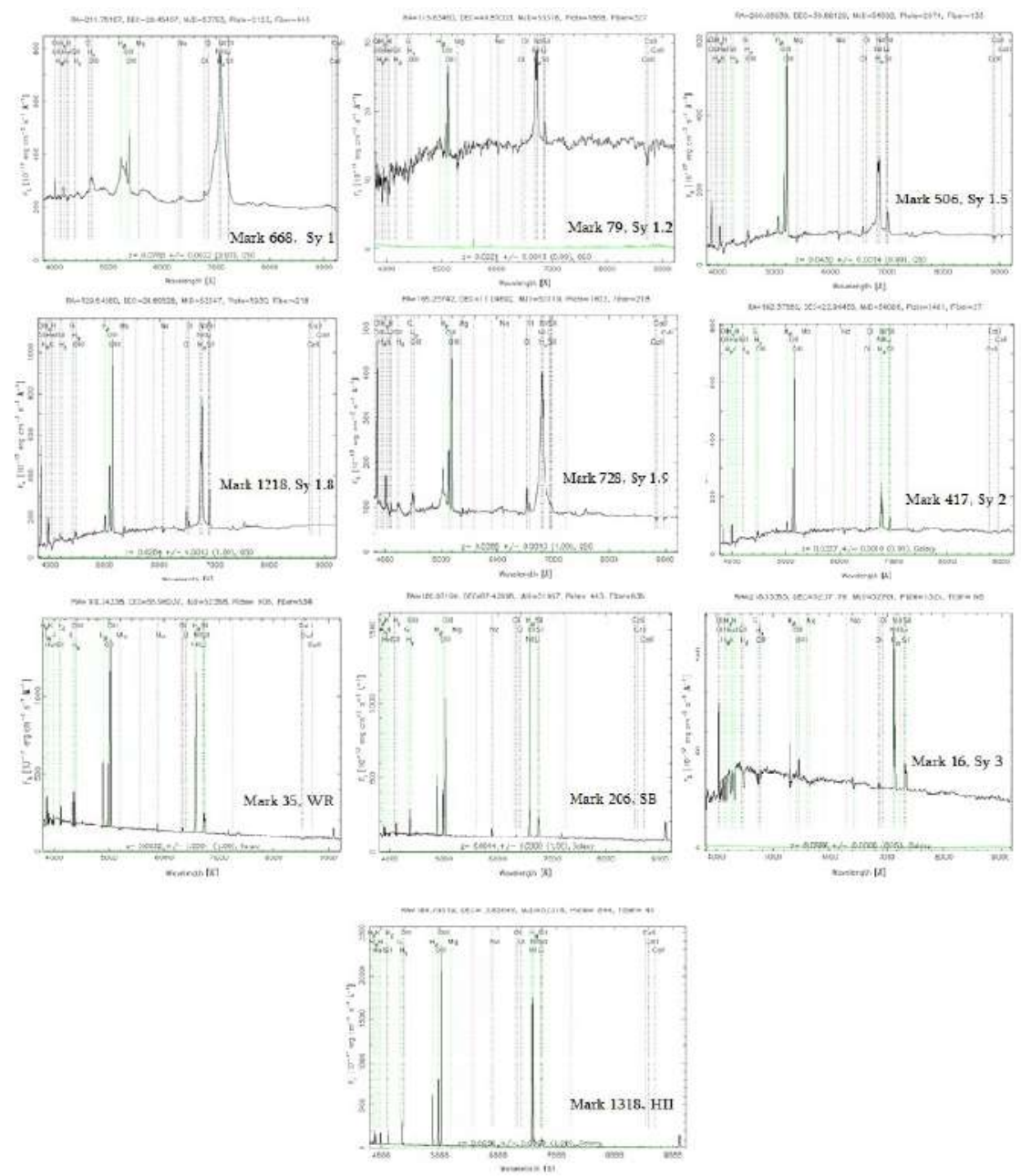

Figure 5: Examples of SDSS spectra of different subsystem type of Mark galaxies

\section{The determination of electron temperatures and oxygen and nitrogen abundances in Mark Galax- ies}

Accurate metallicity play a key role in many investigations of galaxies. Gas-phase oxygen and nitrogen abundances are broadly used to measure these metallicity. It is believed (e.g., Stasinska 2006) that emission lines due to photoionization by massive stars are the most powerful indicator of the chemical composition of galaxies, both in the low- and intermediate redshift universe. Accurate oxygen and nitrogen abundances in H II regions can be derived via the classic Te method, often referred to as the direct method. This method is base on the measurement of the electron temperature t3 
within the $[\mathrm{O}$ III] zone and/or the electron temperature t2 within the $[\mathrm{O}$ II] zone. The ratio of the nebular to auroral oxygen line intensities $[\mathrm{O}$ III $](\lambda$ $4959+\lambda 5007) /[\mathrm{O} \mathrm{III}] \lambda 4363$ is usually used for the $\mathrm{t} 3$ determination, while the ratios of the nebular to auroral nitrogen line intensities $[\mathrm{NII}](\lambda 6548+$ $\lambda 6584) /[\mathrm{N} \mathrm{II}] \lambda 5755$ or $[\mathrm{O} \mathrm{II}](\lambda 3727+\lambda 3729) /[\mathrm{O} \mathrm{II}](\lambda 7320+\lambda 7330)$ are used for the t2 determination. In high-metallicity Hii regions, however, the auroral lines become too faint to be detected.

To select accurate measurements of HII regions in spiral galaxies, we require that the deviation of the oxygen abundance of the HII region from the general linear relation that describes the radial oxygen abundance gradient in the disk to be less than $\sim 0.1$ dex. works. As a result, the abundances from different works are not homogeneous and cannot be directly compared to each other. Therefore, the abundances from different works can be compared and analysed only after those abundances are homogenised, i. e., all the abundances are redetermined in a uniform way. Spectroscopic measurements of H II regions in nearby galaxies were carried out in many.

Spectroscopic measurements of H II regions in nearby galaxies were carried out in many works. The H II regions in one or several galaxies are usually measured and the element abundances are estimated. The different methods for abundance determinations are used in different works.

\section{Star Formation Rate}

Studies of the star formation rate (SFR) in galaxies are important for understanding their formation and evolution. Many indicators for determining the SFR in various parts of the electromagnetic spectrum are currently known. The SFR is widely determined using ultraviolet (UV) continuum emission (Bell \& Kennicutt, 2001), far infrared (FIR) emission (E. F. Bell, 2003) and emission in the radio frequency range (E. F. Bell, 2003), as well as the emission of recombination and forbidden lines in the visible (Moustakas et. al. 2006). One of the most widely used indicators for determining the star formation rate is the $\mathrm{H}_{\alpha}$ line, which is formed in the gaseous medium, after absorption and reprocessing of the emission from young stars beyond the Lyman series limit. It is known that in most galaxies with an ultraviolet excess, the strong UV emission originates mainly in the thermal radiation from young OB-stars. Thus, studies of star formation activity in the nuclei of these galaxies are important.

The results of detailed investigation of physical conditions and chemical abundances about 759 Mark galaxies from SDSS DR7 - DR9 will be published in our extended article. 


\section{References}

French, H. B. 1980, ApJ 240, 41

Goodrich, R. W.; Osterbrock, D. E. 1983, ApJ 269, 416

Heckman, T. M. 1980, Astr. Ap. 87, 152

Huchra, J. P. 1977, ApJS 35, 171

Khachikian, E. E.; Weedman, D. W. 1971, ApJ 7, 389

Markarian, B. E. 1967, Ap 3, 24

Markarian, B. E. 1969, Ap 5, 443

Markarian, B. E.; Lipovetskii, V. A.; Stepanian, J. A, 1981, Ap 17, 321

Osterbrock, D. E. 1976, ApJ 203, 329

Osterbrock, D. E. 1977, ApJ 215, 733

Osterbrock, D. E. 1981, ApJ 249, 462

Osterbrock, D. E.; Dahari, O. 1983, ApJ 273, 478

Weedman, D. W. 1973, ApJ 183, 29

Weedman, D. W.; et al. 1981, ApJ 248, 105

Lee, J., H. ; et al., 2007, Ap. J., 663, 69

Pilygin, L., S., 2010, A\&A, 720, 1738

Ho, L., C.; et al., 1997, Ap. J., 487, 579 